\title{
Standardization of a computerized method for calculating autonomic function test responses in healthy subjects and patients with diabetes mellitus
}

C. Neumann and H. Schmid
Serviço de Endocrinologia, Hospital de Clínicas de Porto Alegre, Universidade Federal do Rio Grande do Sul, 90035-007 Porto Alegre, RS, Brasil

\section{Correspondence \\ H. Schmid \\ Rua Felipe Neri, 296/301 \\ 90440-150 Porto Alegre, RS \\ Brasil \\ Fax: 55 (051) 332-2083}

Research supported by CNPq and FAPERGS.

Received October 6, 1994

Accepted December 5, 1996

\begin{abstract}
The objectives of the present study were 1) to compare results obtained by the traditional manual method of measuring heart rate (HR) and heart rate response (HRR) to the Valsalva maneuver, standing and deep breathing, with those obtained using a computerized data analysis system attached to a standard electrocardiograph machine; 2) to standardize the responses of healthy subjects to cardiovascular tests, and 3) to evaluate the response to these tests in a group of patients with diabetes mellitus (DM). In all subjects (97 healthy and 143 with DM) we evaluated HRR to deep breathing, HRR to standing, HRR to the Valsalva maneuver, and blood pressure response (BPR) to standing up and to a sustained handgrip. Since there was a strong positive correlation between the results obtained with the computerized method and the traditional method, we conclude that the new method can replace the traditional manual method for evaluating cardiovascular responses with the advantages of speed and objectivity. HRR and BPR of men and women did not differ. A correlation between age and HRR was observed for standing $(\mathrm{r}=-0.48, \mathrm{P}<0.001)$ and deep breathing $(\mathrm{r}=$ $-0.41 ; \mathrm{P}<0.002$ ). Abnormal BPR to standing was usually observed only in diabetic patients with definite and severe degrees of autonomic neuropathy.
\end{abstract}

\section{Introduction}

Autonomic neuropathy has been reported in 17 to $40 \%$ of unselected diabetic patients and is associated with a high morbidity and mortality $(1,2)$. Its natural history and the effect of different kinds of treatment proposed for prevention of somatic neuropathy are not known since, with the exception of the Diabetes Control and Complications Trial (DCCT), long-term trials with adequate statistical power to evaluate clinical outcome
Key words

- Diabetes mellitus

- Cardiovascular autonomic neuropathy

- Autonomic tests

- Valsalva maneuver

- Computerized data interpretation 
to criticism and many noninvasive tests have appeared to assess the autonomic nervous function, they are expensive, they were not used in large populations and their sensitivities have not been established. If today we decide to perform an epidemiological study which includes the determination of the cardiovascular autonomic function in diabetic subjects at least one of the tests proposed by Ewing and Clarke must be done, complemented or not with alternative mathematical approaches for measuring heart rate variation $(5,6)$, images with metaiodobenzylguanidine (7) or images obtained with positron emission tomography (8).

The normal results of the tests proposed by Ewing and Clarke, however, might differ between researchers probably because of differences in the maneuvers and equipments (they are not commercially available), and because some techniques are completely computerized whereas others are not. Since the procedure of calculating the responses using the recordings of the electrocardiograph obtained during the maneuvers are tedious and, in order to decrease the variability, they must always be carried out by the same person, they cannot be used for trials conducted for long periods of time and for evaluating many individuals. Some studies define normal responses in populations of subjects without diabetes mellitus (DM) as the minimal value observed (9) whereas others use the 95th or 96th percentile $(10,11)$, the mean/standard deviation (12) or they do not state how normality was established $(13,14)$. These observations and also the recommendations of reseachers in this area $(15,16)$ that standardization of the methods is the first objective in studying cardiovascular autonomic complications of diabetes motivated us to perform the present study. The objectives of the study were 1) to compare results obtained by the traditional method of measuring heart rate (HR) and HRR to the Valsalva maneuver, standing and deep breathing, with those obtained us- ing a computer-based technique developed in our laboratory; 2) to present a standardization of the responses of healthy subjects to autonomic function tests; 3 ) to evaluate the responses of a group of patients with DM to the same tests.

\section{Subjects, Material and Methods}

\section{Subjects}

Healthy subjects. Ninety-seven subjects with normal plasma glucose concentrations who had not been using drugs for at least 30 days were studied. There were 47 women and 64 men, 11 to 67 years old (mean \pm SD: $33.1 \pm 15.4)$.

Diabetic patients. Between March 1991 and 1992, all outpatients with DM attending the Endocrinology Unit, Hospital de Clínicas de Porto Alegre, were screened for inclusion in the study. Those using antihypertensive medication, presenting problems such as alcohol abuse, cardiac rhythm disturbances or acute infectious diseases were excluded. The remaining patients constituted a population of 143 subjects: 86 men and 57 women, 16 to 71 years old (mean \pm SD: $47.6 \pm 13.1$ years); 43 had insulin-dependent DM (IDDM) and 94 had non-insulin-dependent DM (NIDDM).

The clinical characteristics of the diabetic patients are presented in Table 1 .

\section{Material and methods}

An electrocardiograph (FUNBEC: ECG 5 ECAFIX, São Paulo, Brazil) and a microcomputer (APPLE-TK 3000) were used for measuring the heart rate (HR) and HR response (HRR) to 3 maneuvers and they were connected to each other by an interface. The equipment was produced by DEXTER (Porto Alegre, RS, Brazil) with software produced by us. The ECG signals used for calculating the HR were the R-R intervals which were identified by an edge electronic circuit (high 


\begin{tabular}{lcc} 
Table 1 - Clinical characteristics of diabetic patients. & \\
\hline Characteristics & IDDM & NIDDM \\
\hline Number of subjects (total, male/female) & $43(23 / 20)$ & $100(63 / 37)$ \\
Age (mean \pm SD, years) & $31.2 \pm 14.5$ & $54.0 \pm 8.0$ \\
Age (range, years) & $16-50$ & $30-70$ \\
Diabetes treatment (\%) & 100 & 35 \\
$\quad$ Insulin & & 37 \\
$\quad$ Sulfonylurea & & 4 \\
$\quad$ Sulfonylurea plus insulin & & 25 \\
$\quad$ Only diet & $8.7 \pm 60(0.6-23)$ & $8.2 \pm 6.8(0.3-30)$ \\
Duration of diabetes (mean \pm SD; range, years) & & \\
Chronic complications of diabetes & $51.3 / 33.3 / 15.4$ & $57.1 / 36.9 / 5$ \\
$\quad$ Retinopathy (absent/background/proliferative, \%) & $73.8 / 26.2$ & $74.0 / 26.0$ \\
$\quad$ Nephropathy (absent/present, \%) & $46.5 / 18.6 / 18.6 / 16.3$ & $48.0 / 22.0 / 23.0 / 7.0$
\end{tabular}

pass filter). The optimal settings of these parameters were evaluated by one of the researchers by comparing the results of the HRR obtained for one person with the HRR evaluated at the same time by the manual method until the final adjustment was obtained (data not shown). HRR and ECG recordings recognized as QRS complexes (to check the reliability of the detection of the R-R intervals) were obtained from: 1) the screen of the microcomputer, whose software was able to calculate all the responses; 2) the electrocardiograph for measurements using the traditional method (the ratios were calculated by determining the R-R intervals with a measuring tape, always by the same person, who was not informed about the results obtained with the computer program). The software can be obtained by request to H.S.

Blood pressure response (BPR) to two maneuvers was measured with an aneroid sphygmomanometer.

The cardiovascular tests were always performed during the afternoon. The HR, electrocardiographically monitored, was evaluated after $15 \mathrm{~min}$ of resting ( 3 measurements) and before and after deep breathing, the Valsalva maneuver and standing. The tests were performed as follows: a) basal
HR: the subject was asked to lie down and after 15 min the HR was monitored 3 times for 1 min with an interval of 1 min between measurements; b) HRR to deep breathing: the subject breathed deeply and evenly at 6 breaths/min (the examiner informed the subject when each period of $5 \mathrm{~s}$ was ending and whether he must make an inspiratory or expiratory movement). The maximum and minimum heart rate during each breathing cycle was measured and the mean of the differences during three successive breathing cycles was taken as the response; c) HRR to standing: the subject lay quietly with the HR ECG monitored and when the HR was similar to the basal R-R interval, the subject was asked to get up unaided. The ratio between the longest R-R interval (around the 30th beat) and the shortest R-R interval (around the 15th beat) was recorded; d) HRR to the Valsalva maneuver: when the HR was similar to the basal R-R interval the subject blew into a mouthpiece at a pressure of $40 \mathrm{mmHg}$ for $15 \mathrm{~s}$ (the examiner must see jugular engorgement during the test). The ratio between the longest R-R interval shortly after the maneuver and the shortest R-R interval during the maneuver was measured and called the Valsalva ratio; e) BPR to standing: the maneuver was the same as described in c. 
The blood pressure variation when the person was lying down and one minute after standing up was taken as the change in postural blood pressure; f) BPR to sustained handgrip: the maximum voluntary contraction (average of three measurements) was obtained using a handgrip dynamometer (KRATOS, USA) and when the HR of the subject was similar to the basal R-R interval he was asked to maintain the handgrip at $30 \%$ of the maximum voluntary contraction up to a maximum of $5 \mathrm{~min}$; the BPR was measured every minute. The test was repeated if jugular engorgement was observed. The difference between the diastolic blood pressure just before beginning the contraction and the maximal blood pressure during the maneuver was taken as the response.

After defining normal response values for the tests using the third percentile of the results obtained for the healthy group, the patients with diabetes were classified according to the number of abnormal tests: when all of them were normal the patient was considered not to have neuropathy; 1 abnormal test - early involvement; 2 or 3 abnormal tests - definite involvement; 4 or 5 abnormal tests - severe involvement.

\section{Statistical analysis}

The possible effect of age and sex on the responses to these tests was evaluated by regression analysis (Spearman) and the Mann-Whitney test, respectively. The standard used to define normative values was the 97th percentile. Comparisons between the results obtained with the computerized method and the manual method were also made by regression analysis (Spearman). Five percent was considered to be significant in all tests.

\section{Results and Discussion}

The correlation coefficients between the results observed with the computerized and the traditional manual methods were 0.98 , 0.98, 0.99 and 0.99 for the HR (basal rate) and for the HRR to Valsalva maneuver, deep breathing and standing, respectively (Figure 1). These results led us to conclude that for measuring autonomic responses the computerized system is as efficient as the traditional method we had been using. It should be remembered that, when the computerized method is used, subjectivity is eliminated and the time consumed is shorter, i.e., 30 $\min v s 1$ or $1.5 \mathrm{~h}$ for each subject.

The coefficient of variation was calculated after repeating the test 3 to 6 times in the same individual with a 2-3-day interval between tests. The results are the mean of the results obtained with 8 subjects: HRR to Valsalva: 7.4\%; HRR to deep breathing: 17.0\%; HRR to standing: $8.4 \%$; BPR to standing: $246 \%$ and BPR to handgrip: $30.9 \%$. The observation of high coefficients of variation for the same subject in the present study, especially in the tests that evaluated BPR, is in accordance with the results reported by Ewing et al. (12) and Shummer et al. (17).

Figure 2 shows individual cardiovascular responses of healthy subjects to each test relative to age. There was a correlation between age and HRR while the subjects remained standing $(\mathrm{r}=-0.48, \mathrm{P}<0.001)$ and when they took the deep breathing test $(r=$ $-0.41, \mathrm{P}<0.002)$, but not with the responses to the Valsalva maneuver, BPR to sustained handgrip and BPR to standing. Figure 2 also shows that, although the HRR to standing is correlated with age, several of the younger normal subjects had low responses; the third percentile was 1.06 for both the younger and the older people. Regarding the results of the HRR to deep breathing, two groups of subjects can be distinguished: one group of subjects 30 years old or older (97th percentile $=6$ ) and a younger group (97th percentile $=13$ ). In view of these results, it is clear that in order to establish whether HRR to deep breathing is abnormal it is necessary to consider the age of the subject tested. These 

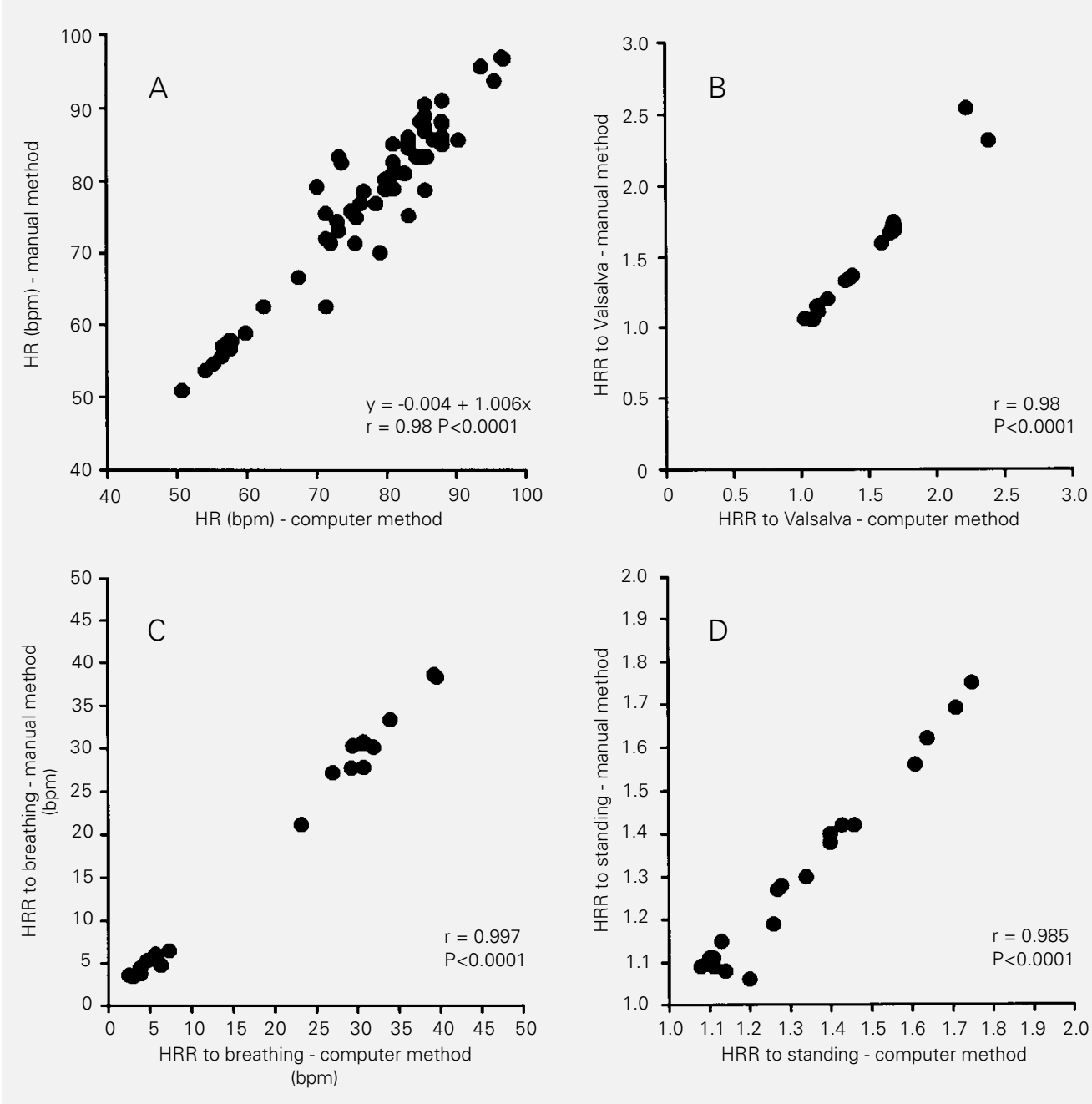

results are similar to those reported by Kennedy et al. (10).

There were no differences between sexes in the results obtained for each test (data not shown) which is in agreement with the report of Ewing et al. (12).

The mean \pm standard deviation, the range and the 97th percentile for the tests performed in healthy subjects are presented in Table 2. For comparison, the values of the mean - 2 standard deviations, and the minimal results observed are also shown. For the purpose of defining the best criteria to establish normal values, the probability of a healthy subject having an abnormal response was calculated. Using the criterion of the minimal response, the result was $0 \%$, exactly as

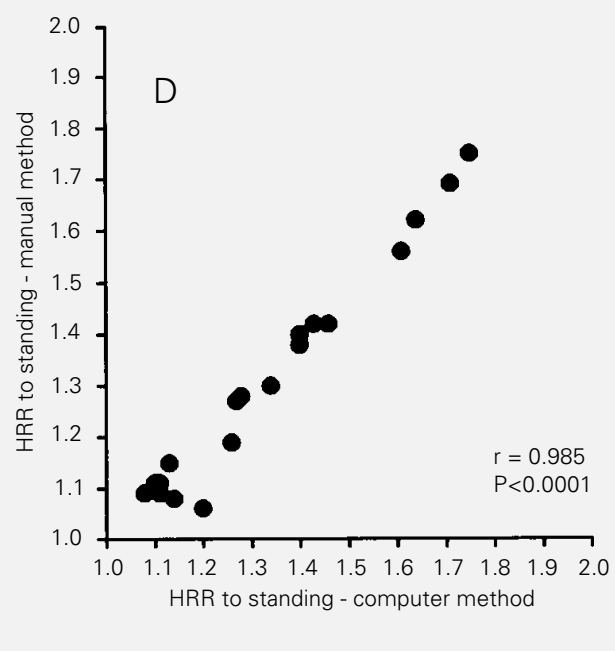

expected, using the criterion of the mean - 2 standard deviations the result was $9.3 \%$ and using the criterion of the 97th percentile the result was $3.1 \%$. Since it is expected that about $2.5 \%$ of a healthy population could have responses lower than the values which are considered normal, it was decided to use the 97th percentile to define normality. The 97th percentiles for the results of the five tests were: 1) deep breathing: for subjects aged 18 to 29 years $=13$; for subjects that were 30 years old or more $=6 ; 2$ ) HRR to standing $=1.06 ; 3)$ HRR to Valsalva $=1.21$; 4) BPR to standing up $=-20 \mathrm{mmHg}$, and 5) BPR to sustained handgrip $=10 \mathrm{mmHg}$.

The mean results obtained for each cardiovascular test in normal subjects, patients
Figure 1 - Comparison of the results calculated by the computer and manual methods for $A$, basal heart rate $(\mathrm{HR})$; $B$, heart rate response (HRR) to the Valsalva maneuver; $C$, heart rate response to deep breathing; $D$, heart rate response to standing up. The results are reported for 20 healthy subjects. Three measurements are reported for $H R$ in panel $A$. 

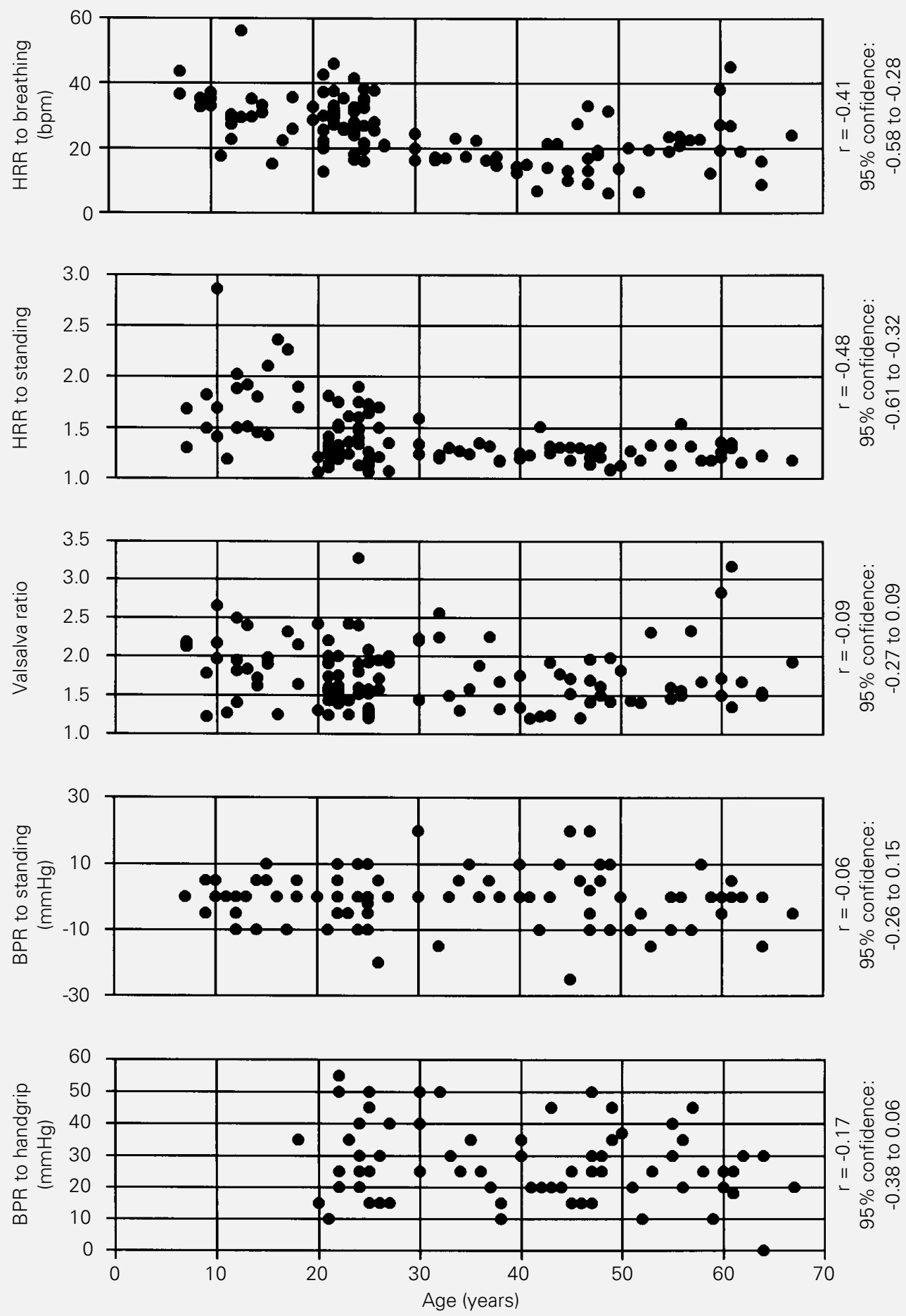

Figure 2 - Heart rate response (HRR) to breathing, to standing and to the Valsalva maneuver and blood pressure responses (BPR) to sustained handgrip and standing as a function of age. Data are reported for 111 healthy subjects and were calculated by the computerized method. 
Table 2 - Results of cardiovascular autonomic tests in normal subjects.

The data were obtained using a computerized data analysis system attached to a standard electrocardiograph machine. HRR, Heart rate response; BPR, blood pressure response; bpm, beats per minute.

\begin{tabular}{lccccc}
\hline Test & $\begin{array}{c}\text { Age range } \\
\text { (years) }\end{array}$ & Mean \pm SD & Median & Range & 97th percentile \\
\hline HRR to the Valsalva maneuver (ratio) & $11-67$ & $1.74 \pm 0.41$ & 1.67 & $1.20-3.28$ & 1.21 \\
HRR to standing (ratio) & $11-67$ & $1.38 \pm 0.26$ & 1.31 & $1.06-2.36$ & 1.06 \\
HRR to deep breathing (bpm) & & & & & \\
$\quad$ under 30 years old & $11-27$ & $28.30 \pm 8.52$ & 29.70 & $9.00-56.10$ & 13.00 \\
$\quad 30$ years old or more & $30-67$ & $19.10 \pm 7.65$ & 18.50 & $6.20-45.00$ & 6.00 \\
BPR to handgrip (mmHg) & $11-67$ & $-0.39 \pm 8.06$ & 0.00 & $-25-20$ & -20.00 \\
BPR to standing (mmHg) & $18-67$ & $27.64 \pm 11.62$ & 25.00 & $0-55$ & 10.00
\end{tabular}

Valsalva ratio

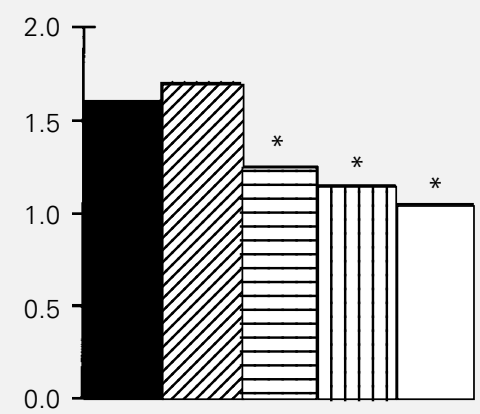

Degree of AN

BPR to standing $(\mathrm{mmHg})$

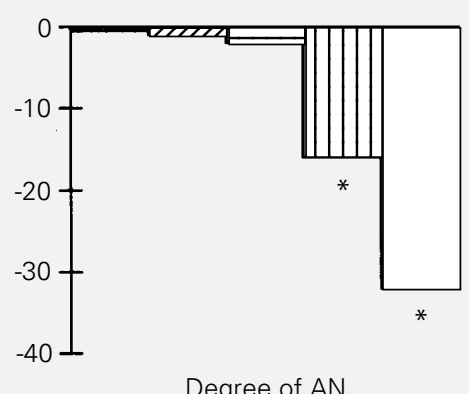

Degree of AN
HRR to standing

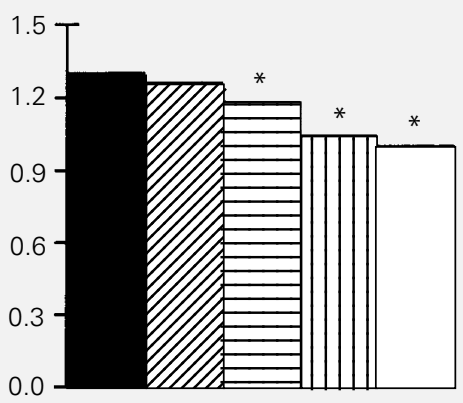

Degree of $A N$

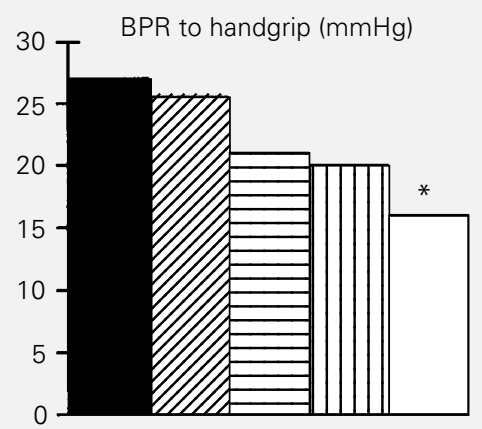

Degree of AN
HRR to breathing (bpm)

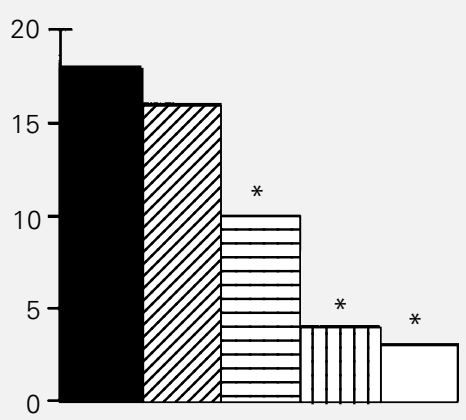

Degree of AN

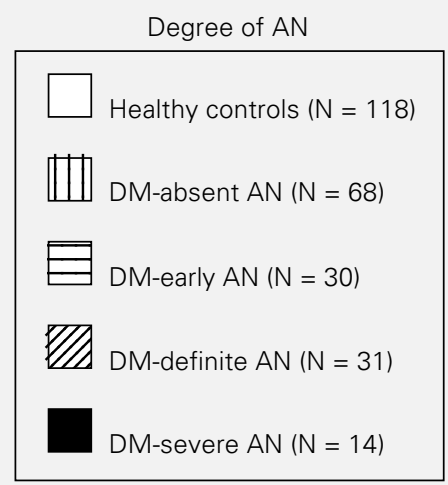

Figure 3 - Responses to cardiovascular tests by healthy controls and diabetic patients with different degrees of autonomic neuropathy (AN). For the deep breathing test, data for persons older than 29 years are reported. The number of persons in each group is given in parentheses. Responses were calculated using the computerized method. HRR, Heart rate response; BPR, blood pressure response. ${ }^{*} \mathrm{P}<0.05$ (Kruskal Wallis one-way analysis of variance). 
Figure 4 - Abnormal results observed in heart rate response (HRR) and blood pressure response (BPR) tests of healthy subjects and diabetic patients with different degrees of autonomic neuropathy (AN). Data are reported as percent of abnormal results for the number of persons in each group indicated within parentheses. Responses were calculated using the computerized method. DM, Diabetes mellitus.

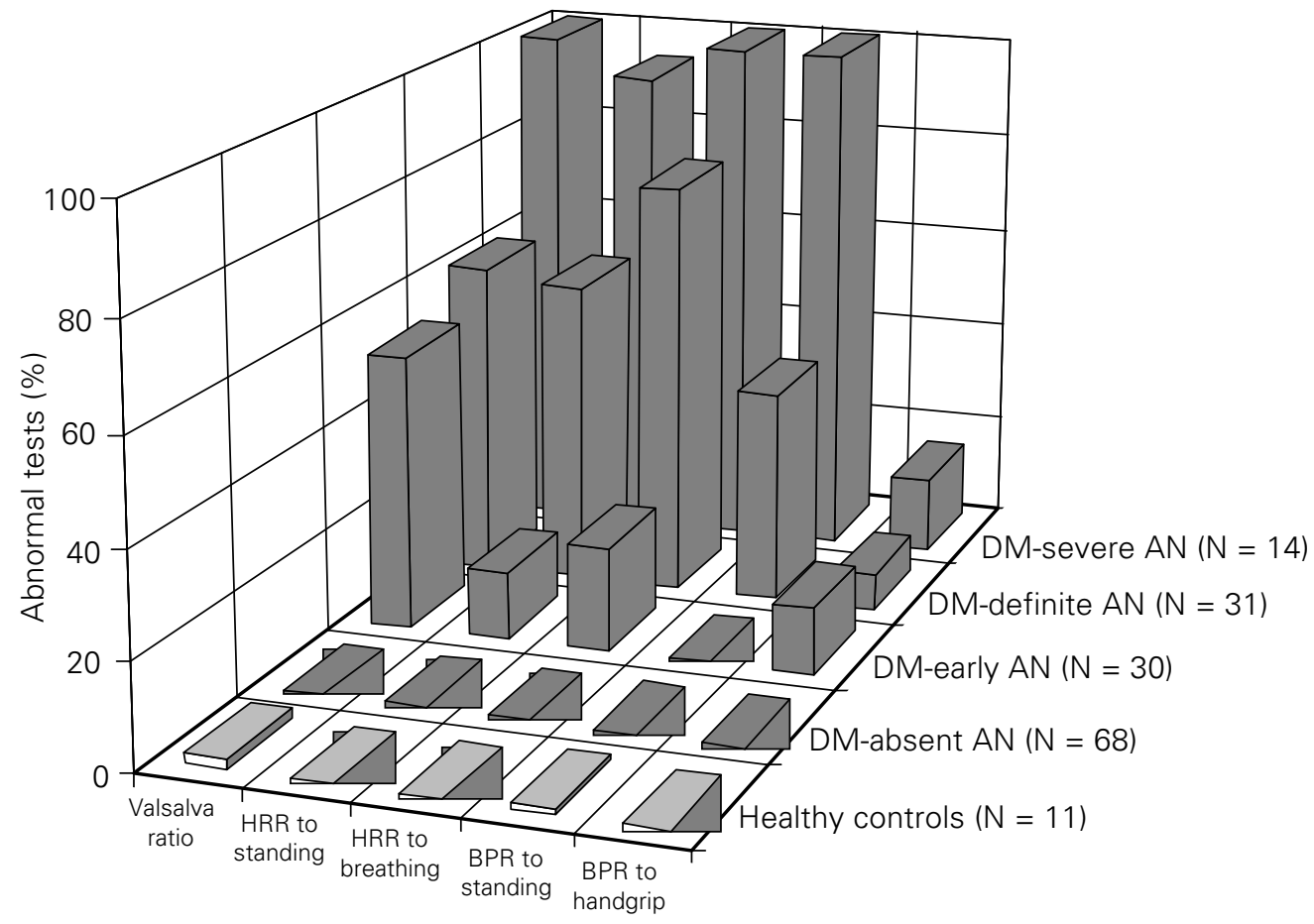

with diabetes without cardiovascular autonomic neuropathy, and patients with diabetes plus three degrees of cardiovascular autonomic neuropathy (incipient, definite and severe) are shown in Figure 3. For all tests it can be seen that the responses decrease progressively as the degree of autonomic dysfunction increases.

The number of abnormal cardiovascular tests in normal subjects, patients with diabetes without cardiovascular autonomic neuropathy and patients with diabetes plus the three degrees of cardiovascular autonomic neuropathy are shown in Figure 4. For the HRR to standing, deep breathing and Valsalva, a significant difference between patients without neuropathy and patients with diabetes and the three degrees of autonomic dysfunction was observed. Differences in HRR between the group with incipient involvement and the group with definite involvement and between the latter and the group with severe autonomic neuropathy were demonstrated for all HRR tests (Figure $3)$. BPR to the sustained handgrip was decreased in the group with severe neuropathy in relation to patients without autonomic neuropathy; differences between the other groups were not observed (Figure 3 ).

Since the only abnormal result observed in $13.0 \%$ of patients with incipient neuropathy was the BPR to sustained handgrip and only $15.4 \%$ of patients with severe neuropathy had an abnormal response to this test (Figure 4), sustained handgrip does not seem to be useful to identify autonomic neuropathy.

The mean BPR to standing was more negative in patients with definite and severe neuropathy compared with all other groups (Figure 3) and $100 \%$ of diabetic patients with severe autonomic neuropathy had an abnormal BPR to standing (Figure 4). Healthy subjects, patients without autonomic neuropathy and with incipient involvement did not differ in their mean response to this test (Figure 3).

We conclude that if a patient has autonomic dysfunction which is not definite or severe, he will usually present at least one abnormal response in the three HRR tests described but the BPR to standing will not be 
altered. In contrast, if a patient with diabetes has postural hypotension but no other problems (such as metabolic decompensation, adrenal failure or other hydroelectrolytic dis- turbances), he probably has a severe dysfunction and his HRR to the Valsalva maneuver, standing and deep breathing may also be abnormal.

\section{References}

1. Ewing DJ, Campbell IW \& Clarke BF (1980). The natural history of diabetic autonomic neuropathy. Quarterly Journal of Medicine, 49: 95-108.

2. Page $M \&$ Watkins $P$ (1978). Cardiorespiratory arrest and diabetic autonomic neuropathy. Lancet, 7: 14-16.

3. Pfeifer MA \& Schumer MP (1995). Clinical trials of diabetic neuropathy: past, present and future. Diabetes, 44: 1355-1361.

4. Consensus statement (1988). Report and recommendations of the San Antonio conference on diabetic neuropathy. Diabetes, 37: 1000-1004.

5. Kinsley BT, Simonson DC, Levy CJ \& Freeman R (1995). Alterations in cardiovascular autonomic function during hypoglycemia assessed by Valsalva maneuver and power spectral analysis. Diabetes, 44 (Suppl 1): 223A.

6. Gelber D, Pfeifer M, Schumer M \& The Statil Study Group (1995). Reliability and comparison of methods to calculate heart rate variability. Diabetes, 44 (Suppl 1): $66 \mathrm{~A}$.

7. Langer $A$, Freeman $M$, Josse $R$ \& Armstrong P (1995). Metaiodobenzylguanidine imaging in diabetes mellitus: assessment of cardiac sympathetic denervation and its relation to autonomic dysfunction and silent myocardial ischemia. Journal of the American College of Cardiology, 25: 610-618.
8. Allman KC, Stevens MJ, Wieland DM, Hutchins GD, Wolfe ER, Greene DA \& Schwaiger M (1993). Noninvasive assessment of cardiac diabetic neuropathy by carbon-11-hydroxyephedrine and positron emission tomography. Journal of the American College of Cardiology, 22: 14251432.

9. Kruter RE, Eizirik DL \& Gross JL (1982). Relationship of the Valsalva ratio to autonomic neuropathy and other complications of diabetes mellitus. Brazilian Journal of Medical and Biological Research, 15: 35-41.

10. Kennedy W, Navarro X, Sakuta M, Mandell H, Knox CK \& Sutherland DER (1989). Physiological and clinical correlates of cardiorespiratory reflexes in diabetes mellitus. Diabetes Care, 12: 399-408.

11. Levin $A B$ (1966). A simple test of cardiac function based upon the heart rate changes induced by Valsalva maneuver. American Journal of Cardiology, 18: 9099.

12. Ewing DJ, Martyn CN, Young RJ \& Clarke BF (1985). The value of cardiovascular autonomic function tests: 10 years experience in diabetes. Diabetes Care, 8: 491498.
13. Jeyarajah R, Samarawickrama P \& Jameel MMM (1986). Autonomic function tests in non-insulin dependent diabetic patients and apparently healthy volunteers. Journal of Chronic Diseases, 39: 479-484.

14. Nathanielsz PW \& Ross EJ (1967). Abnormal response to Valsalva maneuver in diabetics. Relation to autonomic neuropathy. Diabetes, 16: 462-465.

15. Ewing DJ (1990). Which battery of cardiovascular autonomic function tests? Diabetologia, 33: 180-183.

16. Wieling W \& Van Liesshout JJ (1990). The assessment of cardiovascular reflex activity: standardization is needed. Diabetologia, 33: 182-183.

17. Shummer M, Burton G, Burton C, Crum D \& Pfeifer MA (1988). The Diabetes Control and Complications Trial Study Group. Diabetic neuropathy. Part I. Autonomic nervous system data analysis by a computerized central unit in a multicenter trial. American Journal of Medicine, 85 (Suppl 5A): 137-143. 